\title{
Analysis and Optimization of a Synthetic Milkweed Floral Attractant for Mosquitoes
}

\author{
Philip E. Otienoburu • Babak Ebrahimi • \\ P. Larry Phelan • Woodbridge A. Foster
}

Received: 31 May 2011 /Revised: 14 May 2012 / Accepted: 25 May 2012 / Published online: 19 June 2012

(C) The Author(s) 2012. This article is published with open access at Springerlink.com

\begin{abstract}
A pentane extract of flowers of common milkweed, Asclepias syriaca (Asclepiadaceae), elicited significant orientation from both male and female Culex pipiens in a dual-port flight olfactometer. Analysis of the extract by gas chromatography-mass spectrometry revealed six major constituents in order of relative abundance: benzaldehyde, $(E)$ $\beta$-ocimene, phenylacetaldehyde, benzyl alcohol, nonanal, and $(E)$-2-nonenal. Although not all were collected from the headspace profile of live flowers, a synthetic blend of these six compounds, when presented to mosquitoes in the same levels and proportions that occur in the extract, elicited a response comparable to the extract. Subtractive behavioral bioassays demonstrated that a three-component blend consisting of benzaldehyde, phenylacetaldehyde, and (E)-2nonenal was as attractive as the full blend. These findings suggest the potential use of synthetic floral-odor blends for monitoring or control of both male and female diseasevectoring mosquitoes.
\end{abstract}

Keywords Asclepias syriaca $\cdot$ Culex pipiens $\cdot$ Gas chromatography-mass spectrometry (GC-MS) · Milkweed · Nectar feeding · Northern house mosquito · Plant attractants · Subtractive bioassays $\cdot$ Synthetic blends $\cdot$ Asclepidaceae . West Nile virus $\cdot$ Mosquito vector control

P. E. Otienoburu $(\bowtie) \cdot$ B. Ebrahimi $\cdot$ W. A. Foster

Department of Evolution, Ecology \& Organismal Biology,

The Ohio State University,

318W 12th Avenue,

Columbus, OH 43210, USA

e-mail: otienoburu.1@osu.edu

\section{P. L. Phelan}

Department of Entomology, Ohio Agricultural Research and Development Center, The Ohio State University, 1680 Madison Avenue,

Wooster, OH 44691, USA

\section{Introduction}

The northern house mosquito, Culex pipiens L. (Diptera: Culicidae), is a member of a species complex that includes vectors of lymphatic filariasis and several arboviral diseases, such as St. Louis encephalitis (SLE), Rift Valley fever (RFV), and West Nile virus (WNV). In the northeastern quadrant of the United States, C. pipiens recently has become important because of its role in the transmission of WNV. The virus was introduced into New York City in 1999 and is currently the most widespread arboviral disease in the U.S. (CDC, 2010). The blood-feeding pattern of $C$. pipiens (shifting from avian to human feeding within the transmission season) (Edman and Taylor, 1968; Kilpatrick et al., 2006), coupled with its high vector competence (Turell et al., 2005) and ability to carry WNV through the winter, has allowed the virus to spread from its point of origin and maintain transmission throughout the mosquito's range. Thus, C. pipiens is one of the major targets for mosquito vector control and surveillance in the U.S. Current methods of monitoring by abatement districts and health departments include Centers for Disease Control (CDC) light traps and $\mathrm{CO}_{2}$-baited traps, which mainly trap blood-seeking females, along with gravid traps, which target gravid females.

Plant-derived attractants have the potential to act as surveillance-trap lures and, when combined with poisonlaced sugar solutions (Müller et al., 2010), to control mosquito populations. Mosquitoes are attracted to various plant species, from which they obtain nectar and other juices. Sugar feeding by mosquitoes has a significant influence on dispersal (Hocking, 1953; Magnarelli, 1977) and vectorial capacity (Gary and Foster, 2001; Gu et al., 2011), and, contrary to long-held conjecture, is required by both males and females throughout the adult stage (Downes, 1958; Yuval, 1992; Foster, 1995). Both sexes typically first visit 
plants soon after emergence. Males then require sugar at frequent intervals to maintain their energy reserves in order to join nightly mating swarms (Yuval et al., 1992). Females take sugar between blood meals, when they are digesting blood, or when they are gravid (Clements, 1999; Foster, 2008). Furthermore, females of most temperate-climate $\mathrm{Cu}$ lex and Anopheles species enter reproductive diapause in late summer, after which they no longer are attracted to blood hosts, but engage heavily in plant-sugar feeding (Bowen, 1992a).

The potential for plant volatiles to lure mosquitoes has been demonstrated in several laboratory behavioral assays that have used stimuli such as natural plant extracts (Vargo and Foster, 1982; Jepson and Healy, 1988; Mauer and Rowley, 1999) or single floral compounds (Jhumur et al., 2006). In field studies, Bates (1949) reported the successful trapping of anopheline mosquitoes with plant and fruit baits; fruit also proved to be an effective attractant in CDC traps for C. tarsalis (Reisen et al., 1986). Sandholm and Price (1962) observed that mosquitoes in the field were attracted to light-colored flowers with distinct fragrances; odor appears to be primarily responsible for long-range attraction, with visual cues playing a role at shorter range (Thorsteinson and Brust, 1962; Healy and Jepson, 1988; Jepson and Healy, 1988). Orientation to commercially obtained floral extracts and honey has been demonstrated for various mosquito species (Thorsteinson and Brust, 1962; Hancock and Foster, 1997; Foster and Takken, 2004).

Schlein and Müller (2008) and Müller et al. (2008, 2010) reported dramatic population reductions of $C$. pipiens and other mosquito species by spraying a fruit-based sugar bait containing insecticide on vegetation surrounding larval habitats. Light-less CDC traps baited with the blossoms of Tamarix jordanis were highly effective in trapping $C$. pipiens, and populations were reduced where these blossoms were treated with spinosad insecticide (Schlein and Müller, 2008).

Flower species that elicit the highest attraction probably vary by region. During mid-summer in the U.S., mosquitoes have been observed probing blossoms of common milkweed, Asclepias syriaca, at rates disproportionate to their abundance relative to other flowering plants, with one study reporting 54 species occurring in the study area that did not serve as nectar sources (Grimstad and Defoliart, 1974). Another study collected 25 Aedes vexans from one milkweed cluster in just $15 \mathrm{~min}$ (Sandholm and Price, 1962). The observed preference of insects for milkweed flowers, both day and night, possibly is due to its strong and distinctive fragrance, lighter-colored flowers, and greater nectar production (Sandholm and Price, 1962). Common milkweed is indigenous to eastern and midwestern North America, where its principal native pollinators are mainly large Hymenoptera and Lepidoptera (e.g., Jennersten and Morse,
1991). Mosquitoes are incapable of transferring milkweed pollinia and therefore function as nectar thieves, appearing to co-opt the attractive properties used by the pollinators to locate the flowers (Foster, 1995).

Solvent extracts of common milkweed flowers also are attractive to mosquitoes (Vargo and Foster, 1982; Mauer and Rowley, 1999), confirming that volatile chemicals are at least partly responsible for that attraction. Mauer and Rowley (1999) determined the headspace profile of common milkweed flower to be predominantly 2-phenylethanol and benzyl alcohol; however, they found that a synthetic blend of these two compounds was not attractive to C. pipiens. Due to the multiple demonstrations of C. pipiens attraction to milkweed, both in the laboratory and the field, we used a solvent extract of the flower as a potential model for synthetic mosquito lures.

\section{Methods and Materials}

Mosquitoes Experiments were conducted with $C$. pipiens from a colony established in 2009 from larvae collected near Columbus, OH, USA. Larvae were identified at L4 by siphonal hair tufts (Vinogradova, 2000). Adults were maintained in 41-1 clear acrylic cages on a diet of $10 \%$ sucrose, water, and weekly blood meals from the legs of a rooster (ILACUC permit No. 2005A0054). Oviposition water was prepared by soaking grass clippings in aged tap water and allowing fermentation over a 3-d period. Three days after each blood meal, oviposition cups were placed with caged adults, and eggs were collected the following day. Two hundred first-instar larvae were placed into $22.8 \times$ $33.0 \mathrm{~cm}$ aluminum pans with $450 \mathrm{ml}$ of aged tap water. The larvae were provided finely ground TetraMin ${ }^{\circledR}$ flakes, increasing the quantity daily from $50 \mathrm{mg}$ for first instars to $500 \mathrm{mg}$ for final instars until pupae appeared on the 8th and 9 th day post-hatching. Pupae then were counted and transferred to plastic cups and placed in a 41-1 cage supplied with water wicks. Emerging adults were given ad libitum access to water, but were deprived of sugar. Experiments were conducted $36 \pm 12 \mathrm{~h}$ after emergence. The mosquito rearing and maintenance conditions were $27 \pm 1{ }^{\circ} \mathrm{C}, 85 \pm 5 \% \mathrm{RH}$, and 16:8 (L:D), with 30-min gradual crepuscular transitions between photophase and scotophase.

Chemicals Phenylacetaldehyde ( $>90 \%$ ), benzaldehyde ( $\geq 99.5 \%$, purified by redistillation), nonanal ( $\geq 95 \%),(E)$ 2-nonenal $(97 \%)$, and an alkane-standards mixture $\left(\mathrm{C}_{8}\right.$ $\mathrm{C}_{20}$ ) were purchased from Sigma-Aldrich ${ }^{\circledR}$ (St. Louis, MO, USA). Benzyl alcohol (99.9 \%) was purchased from Mallinckrodt Baker, Inc. (Phillipsburg, NJ, USA), and $\beta$ Ocimene $[(75 \%(E)-\beta$-ocimene $)]$ was synthesized by CHEMOS GmbH (Regenstauf, Germany). Synthetics were 
diluted with HPLC-grade $n$-pentane (Fisher Scientific, Pittsburgh, PA, USA).

Extract Preparation Flowers of common milkweed, A. syriaca, were collected in early summer from The Ohio State University campus in Columbus $\left(40^{\circ} 00^{\prime} 18.95^{\prime \prime} \mathrm{N}\right.$, $\left.83^{\circ} 02^{\prime} 47.11^{\prime \prime} \mathrm{W}\right)$, placed in an ice cooler, and transported 10-min to the laboratory. Single florets were plucked from the main milkweed umbel and separated from the calyx and other green parts of the flower, weighed, and placed into a $500 \mathrm{ml}$ narrow-mouth glass Erlenmeyer flask. Milkweed florets were submerged in HPLC-grade $n$-pentane (Fisher Scientific) in a 1:8 ratio $(\mathrm{w} / \mathrm{v})$ for a total solvent volume of $480 \mathrm{ml}$ and held for $24 \mathrm{~h}$ at room temperature, at which time the extract was decanted into $21-\mathrm{ml}$ borosilicate glass vials, capped with Teflon-lined screw caps and stored at $-20{ }^{\circ} \mathrm{C}$.

Headspace Analysis The volatile profile of milkweed flower was characterized by placing a single floret into a $21-\mathrm{ml}$ borosilicate glass vial with a Teflon-lined rubber septum. Volatiles were allowed to equilibrate in the vial headspace at $30{ }^{\circ} \mathrm{C}$ for $10 \mathrm{~min}$ before collections were made. Volatiles were collected by using a divinylbenzene/carboxen/polydimethylsiloxane (DVB/CAR/PDMS) $(50 / 30 \mu \mathrm{m})$ solid phase micro-extraction (SPME) fiber (Supelco, Bellefonte, PA, USA). This mixed-chemistry fiber provides affinity for chemicals with a far broader range of polarity and volatility than PDMS alone. The fiber was introduced to the vial through the septum and exposed for $30 \mathrm{~min}$. The collected volatiles were analyzed immediately by using an Agilent Technologies 6890 series gas chromatograph interfaced to an Agilent Technologies 5973 quadrupole mass selective detector (GC-MS). The SPME fiber was desorbed at $275{ }^{\circ} \mathrm{C}$ for 3 min with a splitless injection onto a Zebron ${ }^{\mathrm{TM}} \mathrm{ZB}-1 \mathrm{~ms}$ column ( $30 \mathrm{~m} \times 0.25 \mathrm{~mm}, 0.25 \mu \mathrm{m}$ film thickness) (Phenomenex, Torrance, CA, USA). The oven temperature was held at $25{ }^{\circ} \mathrm{C}$ for $3 \mathrm{~min}$, then ramped at $15{ }^{\circ} \mathrm{C} / \mathrm{min}$ to $250{ }^{\circ} \mathrm{C}$, where it was held for $2 \mathrm{~min}$. The carrier gas was helium at a flow rate of $1 \mathrm{ml} / \mathrm{min}$. The mass selective detector was operated in EI mode at $70 \mathrm{eV}$, scanning $19-350 \mathrm{~m} / \mathrm{z}$ with a quadrupole temperature of $180{ }^{\circ} \mathrm{C}$ and source temperature of $240{ }^{\circ} \mathrm{C}$.

Extract Analysis To guide the construction of a synthetic floral blend, pentane extracts of milkweed florets were analyzed by GC-MS on a Zebron ${ }^{\mathrm{TM}} \mathrm{ZB}-50$ column $(15 \mathrm{~m} \times$ $0.25 \mathrm{~mm}, 0.25 \mu \mathrm{m}$ film thickness) (Phenomenex), with a 1- $\mu$ l splitless injection at $210{ }^{\circ} \mathrm{C}$. The temperature program began at $25{ }^{\circ} \mathrm{C}$ for $3 \mathrm{~min}$ and increased at $13{ }^{\circ} \mathrm{C} / \mathrm{min}$ to $200{ }^{\circ} \mathrm{C}$, followed by a $25{ }^{\circ} \mathrm{C} / \mathrm{min}$ increase to $280{ }^{\circ} \mathrm{C}$ to remove high-boiling lipids. Other parameters were as described above. Peak identities were validated by re-injection on a Finnegan Trace GC/MS (Thermo Fisher Scientific Inc., Waltham, MA, USA), with a $1: 10$ split at $180{ }^{\circ} \mathrm{C}$ and a Rtx $^{\circledR}$-5MS column (30 $\mathrm{m} \times 0.25 \mathrm{~mm}, 0.25 \mu \mathrm{m}$ film thickness) (Restek, Bellefonte, PA, USA). The helium flow was $1 \mathrm{ml} / \mathrm{min}$, and temperature program was $40^{\circ} \mathrm{C}$ for $1 \mathrm{~min}$, ramped at $15^{\circ} \mathrm{C} / \mathrm{min}$ to $275^{\circ} \mathrm{C}$, with a 2-min final hold time. Retention indices (RI) of compounds were determined on both columns relative to $n$-alkanes $\left(\mathrm{C}_{8}-\mathrm{C}_{20}\right)$. Components of floral headspace and extract were identified by matching their mass spectra to NIST/ EPA/NIH Mass Spectral Library 2005 and comparison of retention indices to published values (Adams, 1995). Identity of major components was confirmed by comparison of spectra and retention times to authentic standards. Peak purity was determined by using AMDIS deconvolution software (NIST, Gaithersburg, MD, USA) to assure the absence of any coeluting components. A synthetic mimic of the extract was constructed by blending authentic standards of the major components identified in the extract. Quantities of each component were individually adjusted until the overall concentration and component proportions closely approximated those of the floral extract (Fig. 1a).

Behavioral Assays All behavioral assays were conducted in a clear acrylic dual-port flight olfactometer, modified from Hancock and Foster (1993), with three main parts: an introduction/release chamber, flight chamber, and trapping ports. The introduction/release chamber was located at the downwind end, measuring $30 \times 40 \mathrm{~cm}$ wide and $30 \mathrm{~cm}$ long. A sliding gate separated the release chamber from the main flight chamber $(30 \times 40 \mathrm{~cm}$ wide, $90 \mathrm{~cm}$ long), which had two cylindrical glass jar trapping ports $(15 \mathrm{~cm}$ long by $7 \mathrm{~cm}$ diameter) on its upwind end. Ports were fitted with borosilicate glass funnels, with the wide end opening into the flight chamber and the narrower ( $3 \mathrm{~mm}$ diam) end pointing into a glass jar. Thus, mosquitoes entering the wide end of the funnel from the flight chamber were channeled into the jar, where they were retained. Trapping ports were located $11 \mathrm{~cm}$ above the flight chamber floor and were separated by $21 \mathrm{~cm}$. The funnel and glass jar were held together by Parafilm ${ }^{\circledR}$ sealing film (Pechiney Plastic Company, Menasha, WI, USA), which was replaced between treatments. We recognized the pitfalls associated with the use of Parafilm ${ }^{\circledR}$ in solvent-based behavioral assays (Millar and Haynes, 1998), and we ensured that this sealant would not come in contact with any chemicals in the olfactometer. A $7.5-\mathrm{mm}$ diam hole in the upwind end of each jar allowed the introduction of purified/humidified air after it had passed from an oil-free air pump through an activated carbon canister, and then through a water column. Air flow was maintained at $50 \mathrm{ml} / \mathrm{s}$ into each port, providing a velocity of $72 \mathrm{~mm} / \mathrm{s}$ in the center of the flight chamber. A black cotton cloth, covering a dampened layer of cotton wool, covered the 
Fig. 1 Total ion chromatograms (a) of florets of milkweed, Asclepias syriaca, pentane extract (broken line), and a synthetic blend (solid line) used to assay upwind attraction of Culex pipiens (Peak \# 1. unknown, 2. (Z)- $\beta$-ocimene, 3. (E)- $\beta$-ocimene, 4 . benzaldehyde, 5. unknown, 6. nonanal, 7. benzyl alcohol, 8. unknown, 9 . phenylacetaldehyde, 10. (E)-2nonenal, 11. 2,6-nonadienal, and 12. phenylethanol; column $=$ Zebron $^{\mathrm{TM}} \mathrm{ZB}-50$ ) and total ion chromatogram (b) of the headspace profile of a single $A$. syraica floret captured on a divinylbenzene/carboxen/polydimethylsiloxane SPME fiber (Peak \# 1. benzaldehyde, 2. myrcene, 3. unknown monoterpene, 4. phenylacetaldehyde, 5 . (Z)- $\beta$-ocimene, 6 . (E)- $\beta$-ocimene, 7. $\gamma$-terpinene, 8. unknown monoterpene, 9. dimethylstyrene, 10. unknown monoterpene, 11. unknown monoterpene, 12. (E,Z)-alloocimene, and 13. (E,E)-alloocimene; column $=$ Zebron $^{\mathrm{TM}} \mathrm{ZB}-1$ )
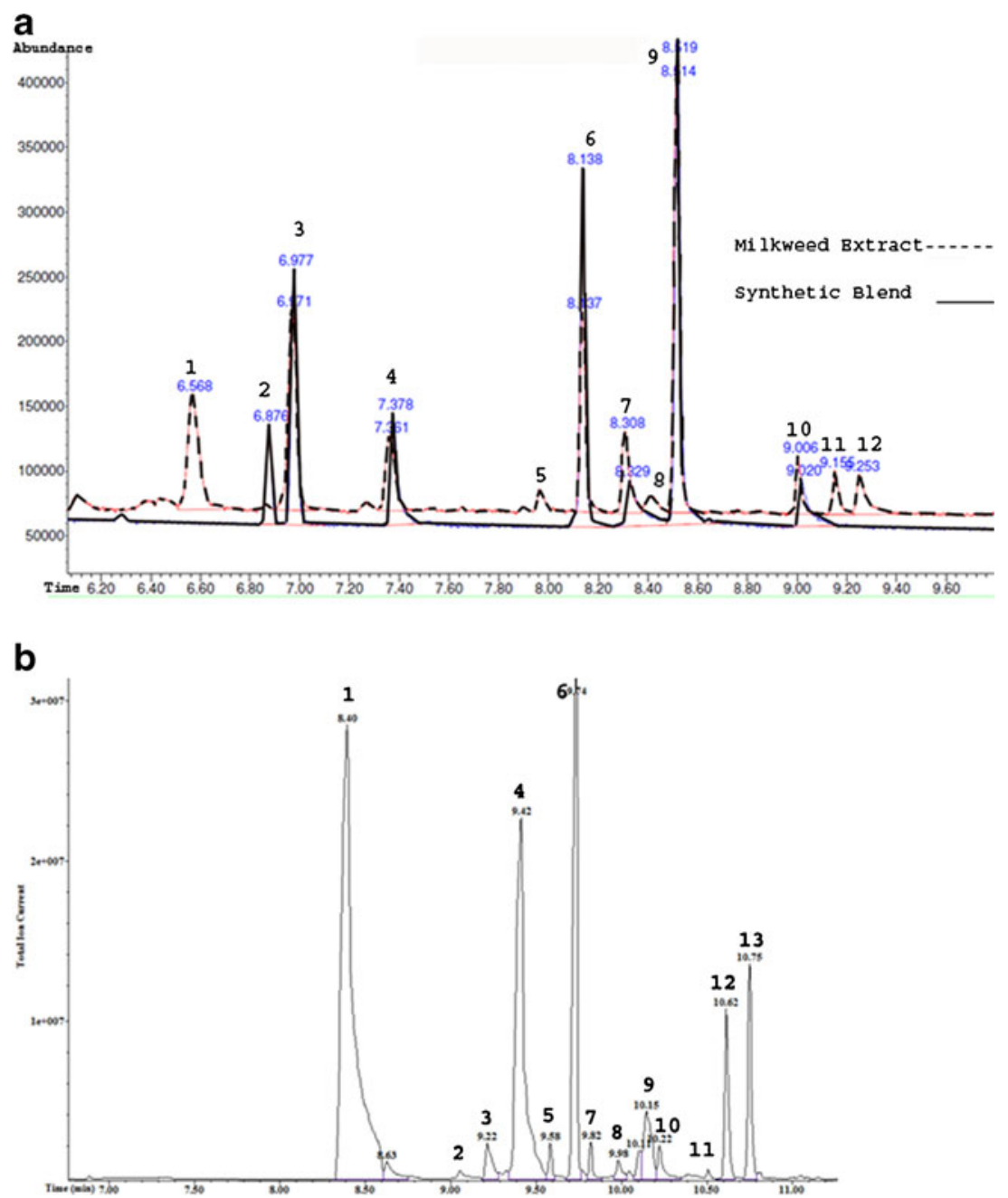

entire floor of the flight chamber, to maintain a level of humidity similar to that within the choice ports. Data loggers $\left(\mathrm{HOBO}^{\circledR}\right.$, Onset Computer Corporation, Bourne, MA, USA) recorded temperature and humidity in the olfactometer, which was 25.0-27.5 ${ }^{\circ} \mathrm{C}$ and 75-95 \% RH. An exhaust duct, connected at the downwind end of the release chamber, directed the effluent air out of the building through an exhaust hood. To test for positional bias of the ports, mosquitoes were released into the chamber after either baiting both ports with honey or leaving both empty. Mosquitoes were divided equally between left and right ports when baited with honey and were found rarely in ports when both were empty.

Response to whole milkweed flowers was determined in the flight olfactometer with $2 \mathrm{~g}$ of florets placed in an aluminum weighing boat in one of the trapping jars, and compared with an empty control jar. Extracts and synthetic chemicals were applied to $15-\mathrm{cm}$ long cotton wicks (TIDI Products, Neenah, WI, USA) and compared to a pentane control. The positions of the treatment and control ports were alternated between bioassays as a further safeguard against positional bias. The olfactometer parts were cleaned with $70 \%$ ethanol followed by water after each experiment; gloves were used at all times to avoid contamination with human-related kairomones. The 16:8 (L:D) light cycle used for mosquito rearing was maintained in the bioassay room. At $2 \mathrm{~h}$ prior to scotophase, approximately 200-300 mosquitoes of both sexes in similar numbers were released through a sleeve connection, directly from an acrylic plastic cage $(30 \times 30 \times 30 \mathrm{~cm})$ into the holding/release chamber, where they were held for $15 \mathrm{~min}$ to acclimatize before the release gate was opened. After $12 \mathrm{~h}$, the numbers of mosquitoes in the treatment port, the control port, and remaining in the flight chamber were recorded. Use of a 12-h period captured the bimodal nocturnal-crepuscular sugar-feeding rhythm of $C$. pipiens, which includes some sugar feeding throughout the night (Yee and Foster, 1992).

Subtractive bioassays were conducted on the synthetic blend to determine the relative activity of the identified components. The full six-component blend was presented in one of the paired olfactometer ports alongside a reducted five-compound blend, in which one of the components was removed. Each reducted blend was tested 4-6 times. After establishing the significance of each chemical on mosquito attraction, we compared the minimal attractive blend against the full six-component blend. This was followed by a dose- 
response study of the minimal blend to determine the optimal concentration for response. Doses were presented in a randomized block design $(N=5)$, with percentage response calculated as the number in the synthetic blend port minus the number in the control port and expressed relative to the number of mosquitoes released.

Video Recording Mosquito flight behavior was recorded by using three infrared RCA closed-circuit video cameras (Lancaster, PA, USA) onto which was mounted a TC1824B

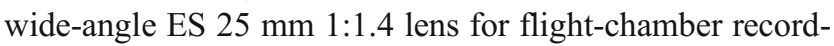
ings or a TC1874C ES $75 \mathrm{~mm} \mathrm{1:1.8} \mathrm{lens} \mathrm{for} \mathrm{each} \mathrm{of} \mathrm{the}$ choice ports. The cameras were controlled in series with a Burle Security Products TC8108 (Lancaster, PA, USA) eight-channel switcher, with video output to two RCA TC1109 video monitors. Video output was recorded with an Emerson EWV404 VCR (Parsippany, NJ, USA) onto VHS tape in time-lapse mode, $20 \mathrm{~s}$ each min.

Statistical Analysis Mosquito response in the flight olfactometer was analyzed by comparing numbers caught in the two ports by a goodness-of-fit chi-squared test with SPSS v. 17 (SPSS Inc, Chicago, IL, USA). The threshold for significance was $\alpha=0.05$. In the dose-response experiment, a regression model of mosquito response to log-transformed blend dose was determined by using the Fitted Line Plot module of Minitab v. 16 (Minitab Inc., State College, PA, USA).

\section{Results}

Chemical Identification Six compounds comprised $>90 \%$ of the relative abundance of the milkweed floret components in the pentane extract: $(E)-\beta$-ocimene, benzaldehyde, nonanal, benzyl alcohol, phenylacetaldehyde, and $(E)$-2-nonenal (Fig. 1a, Table 1). The volatile profile collected by DVB/ CAR/PDMS SPME directly from fresh florets was dominated by three of these compounds: (E)- $\beta$-ocimene, benzaldehyde, and phenylacetaldehyde ( $>75 \%$ of the total), but there were also substantial differences compared to the pentane extract (Fig. 1b). Most notably, (E,Z)- and (E,E)-alloocimene were collected from the flower headspace, but were almost completely absent from the extract, whereas nonanal, a major constituent of floret extract, was missing in the headspace sample. These disparities can be explained partly by variation in volatility among the components, but also likely reflect differences between the chemical composition in flower tissue and what is released. The extract also contained higher-boiling hydrocarbons and fatty acids, but due to their low volatility, they were not included in behavioral studies. Based on the quantities of synthetic standards used to mimic the extract GC profile (Table 1), the total floral concentration of the six major volatiles was estimated at $32 \mu \mathrm{g} / \mathrm{g}$ floret fresh weight.

Flight Olfactometer Response Infrared video recordings showed C. pipiens in the flight chamber engaged in a zigzagging flight that was directed upwind towards ports baited with florets, extracts, or synthetic blends. The angle of the turns progressively decreased as the mosquitoes approached the target. In most cases, mosquitoes flew into the ports, but in a few cases they landed on the entrance of the port and walked in. There was no observed oriented flight towards the control port.

In the first behavioral assay, mosquitoes showed a significant response to whole milkweed florets, where $67 \%$ of released mosquitoes were captured in the floret-baited port, compared to only $6 \%$ in the control port (Fig. $2 \mathrm{a} ; \chi^{2}=515.02$ $d f=1, P<0.001)$. Despite the chemical differences measured between the headspace and extract profiles of milkweed florets, mosquitoes showed a similarly strong response to a pentane extract of milkweed florets (52\%) compared to pentane alone (11\%) (Fig. $\left.2 \mathrm{~b} ; \chi^{2}=61.44, d f=1, P<0.001\right)$. Moreover, mosquitoes were observed in video recordings to probe the extract-treated cotton wick during the early scotophase and early photophase, suggesting that the extract also stimulated a feeding response. This behavior was never observed on control wicks.

Table 1 Major volatile constituents of a pentane extract of the common milkweed, Asclepias syriaca, as determined by gas chromatography-mass spectrometry, and quantities used in a synthetic mimic

\begin{tabular}{llll}
\hline COMPOUND $^{\mathrm{a}}$ & Relative retention index & Diagnostic EI-MS fragment ions (\% intensity) & Synthetic blend $(\mu \mathrm{g} / \mathrm{ml})$ \\
\hline$(E)$ - $\beta$-Ocimene & 1124 & $77(36), 79(42), 91(53), 93(100)$ & $6.14^{\mathrm{c}}$ \\
Benzaldehyde & 1155 & $51(54), 77(100), 105(88), 106(88)$ & 3.27 \\
Nonanal & 1220 & $29(66), 41(100), 56(61), 57(94)$ & 4.14 \\
Benzyl alcohol & 1236 & $77(85), 79(100), 107(59), 108(75)$ & 4.58 \\
Phenylacetaldehyde & 1257 & $65(22), 91(100), 92(24), 120(16)$ & 11.25 \\
$(E)$-2-Nonenal & 1304 & $29(56), 41(100), 55(80), 70(58)$ & 2.12 \\
\hline
\end{tabular}

${ }^{a}$ Identity established by comparison to authentic standard of each compound

${ }^{\mathrm{b}} \mathrm{GC}$ retention times relative to $n$-alkane standards on a Phenomenex ZB-50 column phase

${ }^{\mathrm{c}}$ Synthetic $\beta$-ocimene contained a 3:1 (E:Z) isomer mixture 

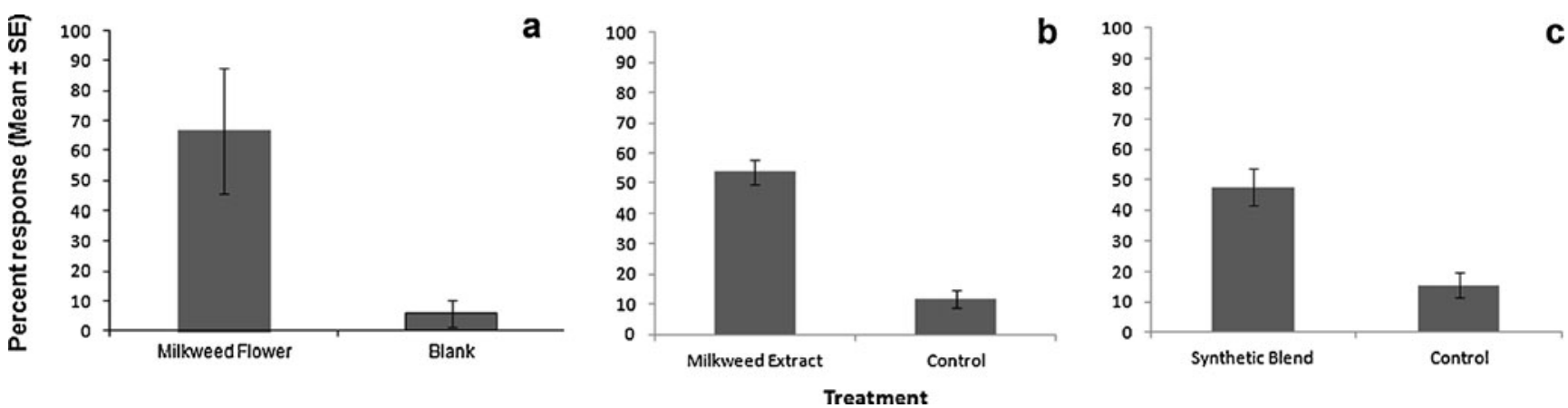

Fig. 2 Percentage of Culex pipiens flying upwind in a dual-port flight olfactometer to: a whole milkweed, Asclepias syriaca, florets; b pentane extract of A. syriaca florets; and (c) synthetic $A$. syriaca floret blend compared against a water a or solvent (b, c) control. *** $P<0.001$ by chi-squared test

Based on the positive response to the milkweed floret extract, a six-component synthetic blend was formulated to simulate the concentrations and relative proportions of major constituents of the pentane extract (Fig. 1a). One discrepancy in the GC profiles was caused by the presence of the (Z)-isomer in synthetic $(E)$ - $\beta$-ocimene, resulting in a synthetic blend that exceeded the levels of this isomer in the floret extract. In the olfactometer, the synthetic floret blend performed similarly to the natural extract, with $48 \%$ of released mosquitoes trapped, compared to $16 \%$ for the control (Fig. $2 \mathrm{c} ; \chi^{2}=120.6, d f=1, P$ $<0.001$ ). Again, video-recordings showed a directed upwind flight response to ports containing the synthetic blend, accompanied by vigorous probing of the treated wicks, but not to the controls. Males and females responded to milkweed floret odors in similar numbers in all three experiments as the sex ratio of mosquitoes captured in either the treatment or control ports did not deviate significantly from 1:1.
The subtractive bioassay of the synthetic blend indicated a significant role for three compounds in C. pipiens response to milkweed: benzaldehyde $\left(\chi^{2}=13.22, d f=1, P<0.001\right)$, phenylacetaldehyde $\left(\chi^{2}=8.25, d f=1, P=0.004\right)$, and $(E)-2$ nonenal $\left(\chi^{2}=3.62, d f=1, P=0.05\right)$ (Fig. 3). When any of these compounds was removed, mosquitoes showed a significant preference for the full blend over the reducted blend. A nearly significant preference was seen for blends missing either $\beta$-ocimene $(P=0.078)$ or benzyl alcohol $(P=0.082)$ compared to the full blend. The activity of benzaldehyde, phenylacetaldehyde, and (E)-2-nonenal was confirmed when the three were combined: the three-component blend captured $31 \%$ of mosquitoes compared to only $7 \%$ for the control (Fig. $4 \mathrm{a} ; \chi^{2}=196.56, d f=1, P<0.001$ ) and was as active as the full blend (Fig. 4b, $4 ; \chi^{2}=3.02, d f=1, P=0.082$ ).

Because quantity as well as quality can determine the intensity of chemically mediated behavior, C. pipiens

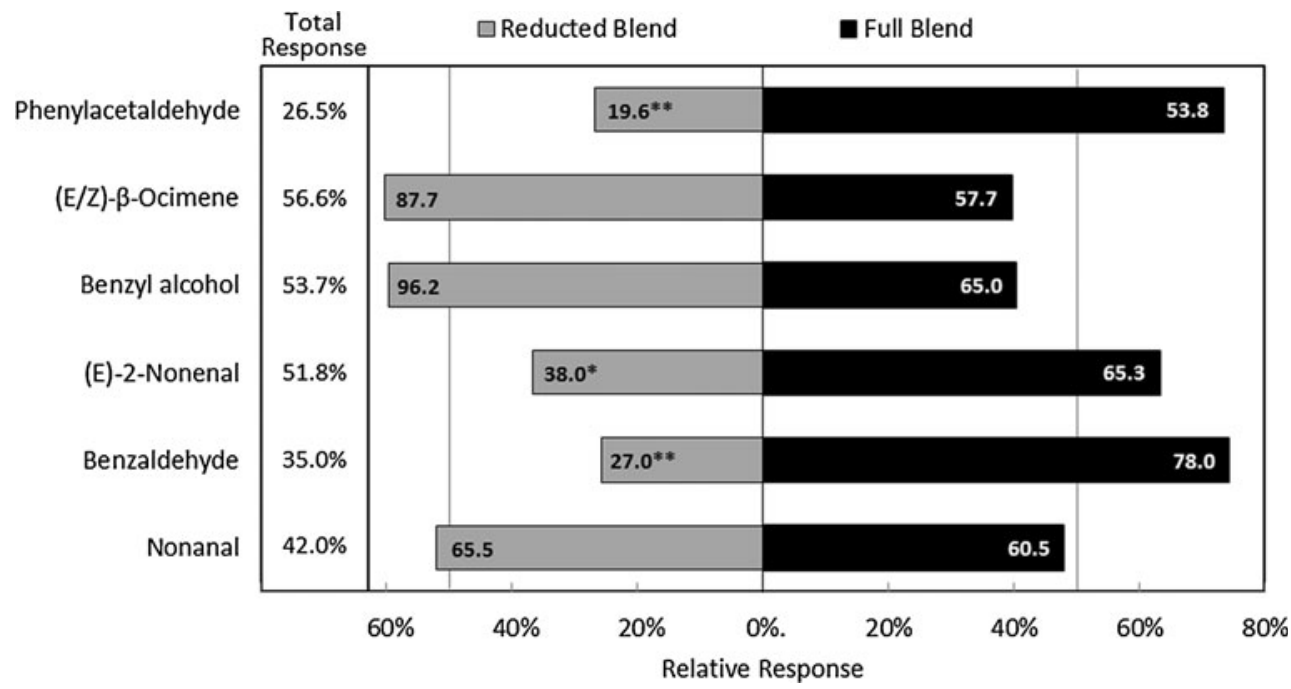

Fig. 3 Choice response of Culex pipiens in a dual-port flight olfactometer to a six-component (full) synthetic blend of milkweed, Asclepias syriaca, floret volatiles compared to blends in which each component has been removed individually (reducted). Total response indicates the percentage of $C$. pipiens released that were captured by one of the blends (no significant differences by 1-way ANOVA).
Relative response shows the proportional response to the reducted (gray bars) and full (black bars) blends. Numbers in bars indicate the mean number of $C$. pipiens responding $(N=4-6)$. Asterisks denote significant differences in mean response to reducted versus full blend by chi-squared test $(* P<0.05, * * P<0.005)$ 
response was measured in the flight olfactometer to different doses of the three-component blend compared to a solvent control. There was a significant positive quadratic response $\left[\log (\right.$ dose $)\left(\mathrm{y}=-20.527 \mathrm{x}^{2}+73.476 \mathrm{x}-43.709 ; R^{2}=57.6 \%\right.$ ], with a response maximum predicted at $62 \mu \mathrm{g}$ (Fig. 5). The response flattened out at both the lowest and highest doses; removal of the two most extreme doses produced a strongerfitting regression model $\left(R^{2}=93.3 \%\right)$, but with a similar predicted optimal dose of $65 \mu \mathrm{g}$.

\section{Discussion}

A positive upwind orientation by $C$. pipiens to floret volatiles of $A$. syriaca was demonstrated in this study, suggesting a new potential source of attractants for use in the field as lures. Males and females showed significant response to milkweed florets, a pentane extract, and a synthetic blend of the extract's six major constituents: benzaldehyde, $(E)$ - $\beta$-ocimene, benzyl alcohol, phenylacetaldehyde, nonanal, and $(E)$-2-nonenal. The first three of these compounds are among the most common floral odor constituents in plants (Knudsen et al., 2006). Subsequent
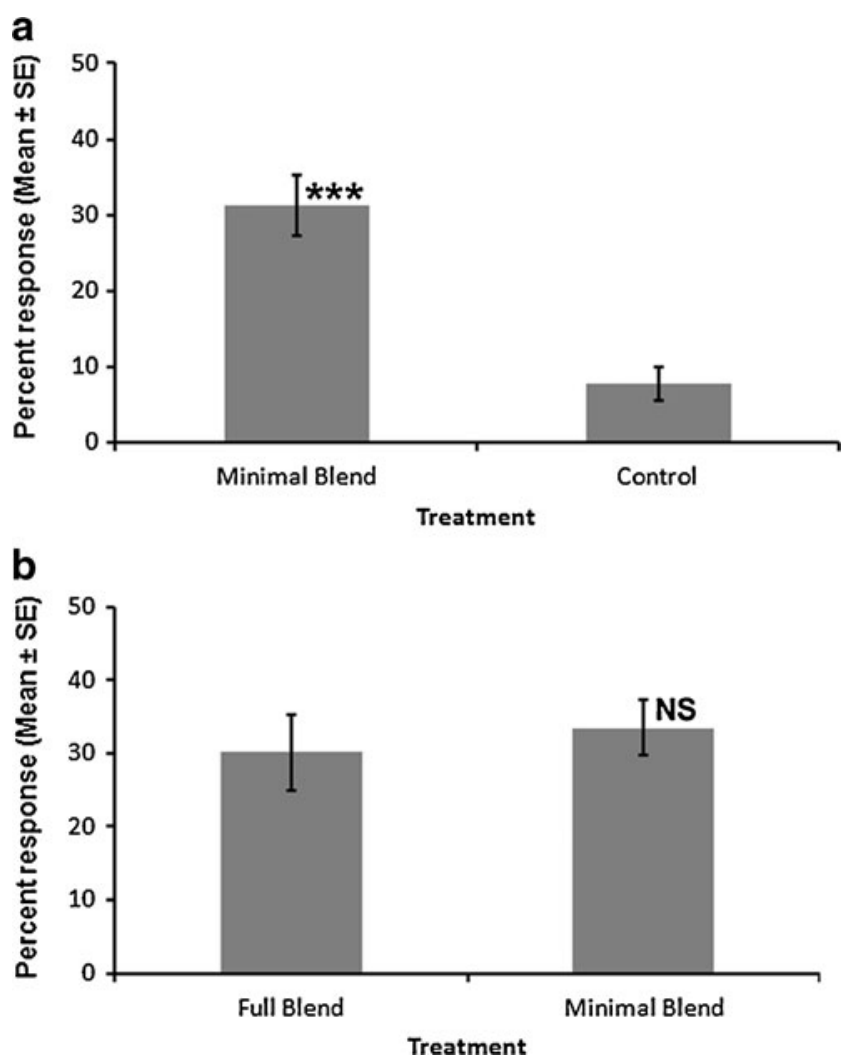

Fig. 4 a Percentage of Culex pipiens flying upwind in a dual-choice flight olfactometer in response to a three-component blend, consisting of benzaldehyde, $(E)$-2-nonenal, and phenylacetaldehyde; *** $P<$ 0.001 by chi-squared test. b Percentage of $C$. pipiens flying upwind in response to the full (six-component) and minimal (three-component) synthetic blends of $A$. syriaca floral odor. NS $=$ no significant difference

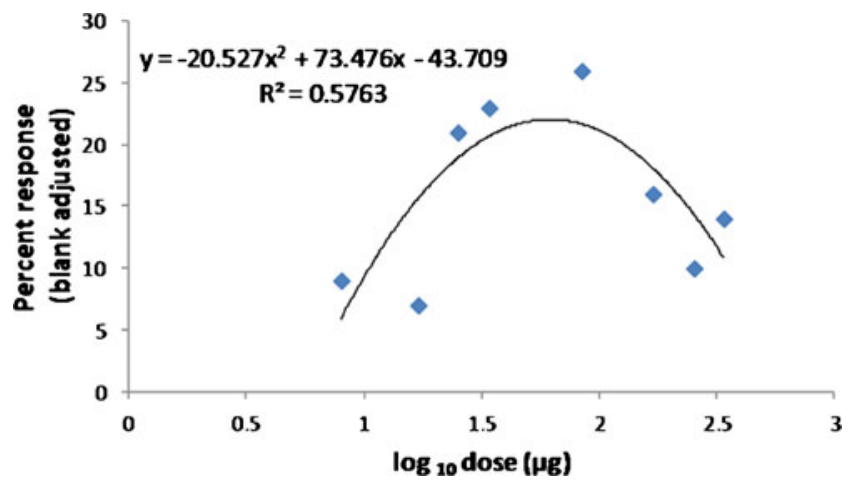

Fig. 5 Percentage of Culex pipiens flying upwind in a dual-choice flight olfactometer in response to different concentrations of a threecomponent kairomone blend (benzaldehyde, $(E)$-2-nonenal, and phenylacetaldehyde) after subtracting the response to a paired solvent (pentane) control

subtractive bioassays of the synthetic blend demonstrated that only three of its components contributed to attraction: benzaldehyde, phenylacetaldehyde, and (E)-2-nonenal.

The attraction of mosquitoes to plant odors has been demonstrated in numerous field and laboratory studies, but few studies have shown significant attraction to synthetics. Mauer and Rowley (1999) observed orientation of $C$. pipiens to a methylene chloride extract of $A$. syriaca in a dual port olfactometer. However, a synthetic blend of the two dominant compounds in the extract headspace, benzyl alcohol and 2phenylethanol, failed to attract the mosquitoes. We did not test 2-phenylethanol, but benzyl alcohol was not active in our flight olfactometer bioassays. Jhumur et al. (2007) also found benzyl alcohol to not be very attractive to $C$. pipiens var. molestus, although Puri et al. (2006) recorded significant upwind response by $C$. quinquefasciatus to the compound.

Flowers of Tanacetum vulgare also attract Culex species in the field (Andersson and Jaenson, 1987), and Bowen (1992b) discovered a high proportion of both broadly- and narrowlytuned antennal receptor neurons of $C$. pipiens sensitive to thujone, the primary constituent of $T$. vulgare fragrance. However, thujone only elicited a close-range dose-dependent probing response in C. pipiens; it did not stimulate upwind flight in a wind-tunnel olfactometer (Bowen, 1992b). The only previous flower-based synthetic blend causing mosquito orientation was developed from Silene otites, whose flowers attract mosquitoes in the field (Brantjes and Leemans, 1976). The synthetic blend mimic consisted of phenylacetaldehyde, veratrole, and 2-methoxyphenol, the first of which elicited the strongest attraction in C. pipiens var. molestus when presented individually (Jhumur et al., 2006). Phenylacetaldehyde also was a prominent component of our $A$. syriaca headspace and solvent extract, and it was essential for maximum attraction to the synthetic blend.

Although nonanal was a major component of $A$. syriaca floret extract, we saw no evidence that it played a role in 
attraction in our bioassays. In contrast, from single-cell recordings, Syed and Leal (2009) determined that ca. $40 \%$ of all antennal olfactory receptors of $C$. quinquefasciatus were acutely sensitive to nonanal, and it produced a synergistic effect on trap catch when presented with $\mathrm{CO}_{2}$. This apparent discrepancy likely is explained by differences in mosquito physiological state. Nonanal is a major skin odorant of birds and mammals, including humans, resulting from the oxidation of sebaceous fatty acids (Haze et al., 2001). This fact, along with the significant behavioral interaction with $\mathrm{CO}_{2}$, suggests that nonanal may play an important role in mediating Culex host-finding, but does not attract sugar-seeking mosquitoes.

We found notable differences between profiles of the pentane extract and the SPME-collected headspace of $A$. syriaca florets. Pentane extracts were dominated by phenylacetaldehyde, $(E)$ - $\beta$-ocimene, and nonanal, whereas the headspace profile contained primarily benzaldehyde, ocimene isomers, and phenylacetaldehyde. Disparities between extracts and chemicals collected in the headspace might be explained by differences in the physico-chemical properties of the constituents, deep penetration by solvents to extract compounds that are not normally released by the tissue, and/or selective trapping of chemicals by SPME. In this study, differences between the methods are largely consistent with chemical differences in vapor pressure. Benzaldehyde, $\beta$-ocimene, and alloocimene have the highest vapor pressures of all the chemicals identified from milkweed, and they make up most of the volatile profile. The vapor pressure of benzaldehyde is almost twice that of nonanal, almost four times that of $(E)$-2-nonenal, and more than six times that of benzyl alcohol. The latter three compounds were either absent or found in very low levels in the headspace analysis. Phenylacetaldehyde is intermediate in its volatility, but was also the component found in the highest levels in the pentane extract.

Volatile collection analyses can produce misleading results, as they may not accurately reflect the actual proportionality of constituents in the headspace. We do not believe that this was a major factor for explaining the differences in the headspace and extract profiles of milkweed due to our choice of SPME phase. Although PDMS has been the most widely used SPME phase, it is actually a poor choice for characterizing plant volatile profiles that contain constituents with a range of volatilities and functional groups. In preliminary studies (not shown), we found a broader array of volatile compounds was trapped from various flowers by DVB/CAR/PDMS compared to PDMS alone. This mixed-bed fiber not only employs a broader range of phase polarity, but also incorporates both adsorption and partitioning as mechanisms of collection (Koziel and Novak, 2002). A number of recent studies have demonstrated the higher recovery efficiency and linearity by DVB/CAR/PDMS compared to other SPME phases for all of the compound classes that we identified from milkweed (Cui et al., 2009; Ferreira et al., 2009; Zhang et al., 2009). For example, Zhang et al. (2009) found that volatile profiles of longan fruit extracted by a $50 / 30 \mu \mathrm{m}$ DVB/CAR/PDMS fiber were $3 \mathrm{x}$ higher in terpenes, $5 \mathrm{x}$ higher in alcohols, and $14 \mathrm{x}$ higher in esters than those produced by a $100 \mu \mathrm{m}$ PDMS fiber, and revealed volatile carbonyls and acids, which were completely absent from PDMS profiles.

This study points to floral odors as a potential new model for chemical lures useful for mosquito sampling or control. If similar attraction can be demonstrated at the field level, as has already been demonstrated with human kairomone blends (Mukabana et al., 2012), synthetic floral blends could potentially be used in trapping devices to sample adult populations. Relative to animal-derived odors (Mukabana et al., 2012), floral odors have the advantage of attracting both sexes of mosquitoes in proportional numbers, and females in all gonotrophic states and in reproductive diapause. Given that $C$. pipiens visits a variety of flowers for nectar-feeding, the three compounds identified here are likely not the only floral components attractive to them, and more effective blends may remain to be discovered. It also remains to be seen whether different mosquito species use similar chemical cues. Future research should seek additional attractants and determine optimal blend release rates, delivery systems, and trap designs for maximizing capture in the field.

Acknowledgments We thank Robert Aldridge for help in rearing mosquitoes and Ephantus Muturi for suggestions on an early draft of this manuscript. This research was supported through NIH grant R01AI064506.

Open Access This article is distributed under the terms of the Creative Commons Attribution License which permits any use, distribution, and reproduction in any medium, provided the original author(s) and the source are credited.

\section{References}

ADAMS, R. P. 1995. Identification of Essential Oil Components by Gas Chromatography/Mass Spectroscopy. Allured Publishing, Carol Stream, IL.

ANDERSSON, I. H. and JAENSON, T. G. T. 1987. Nectar feeding by mosquitoes in Sweden with special reference to Culex pipiens and Cx torrentium. Med. Vet. Entomol. 1:59-64.

BATES, M. 1949. pp. 379, The natural history of mosquitoes. The Macmillan Co., New York, NY.

Bowen, M. F. 1992a. Patterns of sugar feeding in diapausing and nondiapausing Culex pipiens (Diptera: Culicidae) females. $J$. Med. Entomol. 29:843-849.

Bowen, M. F. 1992b. Terpene-sensitive receptors in female Culex pipiens mosquitoes: Electrophysiology and behaviour. J. Insect Physiol. 38:759-764.

Brantjes, N. B. M. and Leemans, J. A. A. M. 1976. Silene otites (Caryophyllaceae) pollinated by nocturnal Lepidoptera and mosquitoes. Acta Bot. Neerl. 25:281-295.

Centers for Disease Control (CDC). 2010. Surveillance for Human West Nile Virus Disease-United States, 1999-2008. MMWR CDC Surveill Summ 59(No. SS-2) [April 2, 2010]. 
Clements, A. N. 1999. The biology of mosquitoes. Sensory reception and behavior. CABI Publishing, New York, NY. 740 p.

CUI, S., TAN, S., OUYANG, G., JiAnG, S., and PAWliszYN, J. 2009. Headspace solid-phase microextraction gas chromatographymass spectrometry analysis of Eupatorium odoratum extract as an oviposition repellent. J. Chromatogr. B 877:1901-1906.

Downes, J. A. 1958. The feeding habits of biting flies and their significance in classification. Annu. Rev. Entomol. 3:249-266.

EDMAN, J. D. and TAYLOR, D. J. 1968. Culex nigripalpus: Seasonal shift in the bird-mammal feeding ratio in a mosquito vector of human encephalitis. Science 161:67-68.

Ferreira, L., Perestrelo, R., Caldeira, M., and CÂmara, J. S. 2009. Characterization of volatile substances in apples from Rosaceae family by headspace solid-phase microextraction followed by GC-qMS. J. Sep. Sci. 32:1875-1888.

FOSTER, W. A. 1995. Mosquito sugar feeding and reproductive energetics. Annu. Rev. Entomol. 40:443-474.

Foster, W. A. 2008. Phytochemicals as population sampling lures. $J$. Am. Mosq. Control Assoc. 24:138-146.

Foster, W. A. and TAKKEN, W. 2004. Nectar-related vs. humanrelated volatiles: behavioural response and choice by female and male Anopheles gambiae (Diptera: Culicidae) between emergence and first feeding. Bull. Entomol. Res. 94:145-157.

GARY, R. E. and FosteR, W. A. 2001. Effects of available sugar on the reproductive fitness and vectorial capacity of the malaria vector Anopheles gambiae (Diptera: Culicidae). J. Med. Entomol. 38:22-28.

Grimstad, P. R. and Defoliart, G. R. 1974. Nectar sources of Wisconsin mosquitoes. J. Med. Entomol. 11:331-341.

Gu, W., Müller, G., Schlein, Y., NovaK, R. J., and Beier, J. C. 2011. Natural plant sugar sources of Anopheles mosquitoes strongly impact malaria transmission potential. PLOS ONE 6:e15996.

HANCOCK, R. G. and Foster, W. A. 1993. Effect of preblood-meal sugar on sugar seeking and upwind flight by gravid and parous Aedes aegypti (Diptera: Culicidae). J. Med. Entomol. 30:353-359.

HANCOCK, R. G. and FOSTER, W. A. 1997. Larval and adult nutrition effects on the blood/nectar choice of Culex nigripalpus mosquitoes. Med. Vet. Entomol. 11:112-122.

Haze, S., Gozu, Y., NaKamura, S., Kohno, Y., Sawano, K., Ohta, H., and YAMAZAKIET, K. 2001. 2-Nonenal newly found in human body odor tends to increase with aging. J. Invest. Dermatol. 116:520-524.

HeAly, T. P. and JePSON, P. C. 1988. The location of floral nectar sources by mosquitoes: the long-range responses of Anopheles arabiensis Patton (Diptera: Culicidae) to Achillea millefolium flowers and isolated floral odour. Bull. Entomol. Res. 78:651-657.

HockING, B. 1953. The intrinsic range and speed of flight of insects. Trans. Roy. Entomol. Soc. London 104:223-346.

JenNERSTEN, O. and MORSE, D. H. 1991. The quality of pollination by diurnal and nocturnal insects visiting common milkweed, Asclepias syriaca. Am. Midland. Nat. 125:18-28.

JePSON, P. C. and HeALY, T. P. 1988. The location of floral nectar sources by mosquitoes: an advanced bioassay for volatile plant odours and initial studies with Aedes aegypti (L.) (Diptera: Culicidae). Bull. Entomol. Res. 78:641-650.

Jhumur, U. S., DötTerl, S., and JÜrgens, A. 2006. Naïve and conditioned responses of Culex pipiens pipiens Biotype molestus (Diptera: Culicidae) to flower odors. J. Med. Entomol. 43:1164-1170.

JHUMUR, U. S., DöTTERL, S., and JÜRGENS, A. 2007. Electrophysiological and behavioural responses of mosquitoes to volatiles of Silene otites (Caryophyllaceae). Arthropod-Plant Inte. 1:243-254.

KilPATRICK, A. M., Kramer, L. D., Jones, M. J., MARRA, P. P., and DASZAK, P. 2006. West Nile virus epidemics in North America are driven by shifts in mosquito feeding behavior. PLoS Biol. 4:06060610.

KNUdSEn, J. T., ERIKSSON, R., GERShenZON, J., and StÅHL, B. 2006. Diversity and distribution of floral scent. Bot. Rev. 72:1-120.
KozIEL, J. A. and NovAK, I. 2002. Sampling and sample-preparation strategies based on solid-phase microextraction for analysis of indoor air. Trends Anal. Chem. 21:840-850.

Magnarelli, L. A. 1977. Nectar feeding by Aedes sollicitans and its relation to gonotrophic activity. Environ. Entomol. 6:237-242.

MAUER, D. J. and Rowley, W. A. 1999. Attraction of Culex pipiens pipiens (Diptera: Culicidae) to flower volatiles. J. Med. Entomol. 36:503-507.

Millar, J. G. and Haynes, K. F. (EDS.) 1998. pp. 390, Methods in chemical ecology: Chemical methods. Kluwer Academic Publishers, Norwell, MA.

Mukabana, W. R., Mweresa, C. K., Otieno, B., Omusula, P., SMALlEGANGe, R. C., VAN LOON, J., and TAKKEN, W. 2012. A Novel synthetic odorant blend for trapping of malaria and other African mosquito species. J. Chem. Ecol. 38:235-244.

Müller, G. C., KRAVChenKo, V. D., and SchleIN, Y. 2008. Decline of Anopheles sergentii and Aedes caspius populations following presentation of attractive toxic (Spinosad) sugar bait stations in an oasis. J. Am. Mosq. Control Assoc. 24:147-149.

MÜLleR, G. C., JunNILA, A., and SCHLEIN, Y. 2010. Effective control of adult Culex pipiens by spraying an attractive toxic sugar bait solution in the vegetation near larval habitats. J. Med. Entomol. 47:63-66.

Puri, S. N., Mendki, M. J., Sukumaran, D., Ganesan, K., Prakash, S., and SeKHAR, K. 2006. Electroantennogram and behavioral responses of Culex quinquefasciatus (Diptera: Culicidae) females to chemicals found in human skin emanations. $J$. Med. Entomol. 43:207-213.

Reisen, W. K., Meyer, R. P., AND Milby, M. M. 1986. Patterns of fructose feeding by Culex tarsalis (Diptera: Culicidae). J. Med. Entomol. 23:366-373.

Sandholm, H. A. AND PRICE, R. D. 1962. Field observations on the nectar feeding habits of some Minnesota mosquitoes. Mosq. News 22:346-349.

SChleIN, Y. and MÜLleR, G. C. 2008. An approach to mosquito control. Using the dominant attraction of flowering Tamarix jordanis trees against Culex pipiens. J. Med. Entomol. 45:384-390

SYED, Z. and LEAL, W. 2009. Acute olfactory response of Culex mosquitoes to a human- and bird-derived attractant. Proc. Natl. Acad. Sci. USA 106:18803-18808.

Thorsteinson, A. J. and BRUST, R. A. 1962. The influence of flower scents on aggregations of caged adult Aedes aegypti. Mosq. News $22: 349-352$

Turell, M. J., Dohm, D. J., Sardelis, M. R., O'Guinn, M. L., READIS, T. G., AND BLOW, J. A. 2005. An update on the potential of North American mosquitoes (Diptera: Culicidae) to transmit West Nile virus. J. Med. Entomol. 42:57-62.

VARGO, A. M. and Foster, W. A. 1982. Responsiveness of female Aedes aegypti (Diptera: Culicidae) to flower extracts. J. Med. Entomol. 19:710-718.

VinogradovA, A. B. 2000. Culex pipiens pipiens mosquitoes: Taxonomy, distribution, ecology, physiology, genetics, applied importance and control. Pensoft Publishers, Sofia, Bulgaria. 250 p.

Yee, W. L. and Foster, W. A. 1992. Diel sugar-feeding and hostseeking rhythms in mosquitoes (Diptera: Culicidae) under laboratory conditions. J. Med. Entomol. 29:784-791.

Yuval, B. 1992. The other habit: Sugar feeding by mosquitoes. Bull. Soc. Vector Ecol. 17:150-156.

Yuval, B., Holliday-Hanson, M. L., and Washino, R. K. 1992. Energy budget of swarming male mosquitoes. Ecol. Entomol. 19:74-78.

Zhang, Y., GaO, B., Zhang, M., ShI, J., and XU, Y. 2009. Headspace solid-phase microextraction-gas chromatography-mass spectrometry analysis of the volatile components of longan (Dimocarpus longan Lour.). Eur. Food Res Technol. 229:457-465. 